\title{
Tone chroma is functional in melody recognition
}

\author{
HOWARD J. KALLMAN and DOMINIC W. MASSARO \\ University of Wisconsin, Madison, Wisconsin 53706
}

\begin{abstract}
Musical notes can be defined both in terms of their fundamental frequencies (tone height) and their positions in the musical octave (tone chroma). Although there is some evidence indicating that both tone height and chroma can contribute to tone perception, Deutsch (1969, 1972) suggested that chroma is not functional in melody recognition. By systematically transforming melodies so as to preserve one or another type of information, Idson and Massaro (1978) demonstrated that chroma was also functional in melody recognition. However, in the Idson and Massaro study, subjects repeatedly heard the same melodies and were asked to identify them out of a limited set of possibilities (forced-choice procedure). The repetition of melodies and the forced-choice procedure might have encouraged subjects to develop special recognition strategies. To preclude this possibility, subjects in the present experiments heard each melody only once and were not informed in advance of the possible melodies. When systematic transformations similar to those used by Idson and Massaro were applied to familiar melodies, similar results were found. Therefore, it is unlikely that special recognition strategies were responsible for the results of the Idson and Massaro (1978) study: tone chroma contributes to melody recognition in both types of experimental procedures. The functional role of tone chroma in melody recognition is consistent with the idea that octave generalization not only applies to the perception of single tones but also occurs in the perception of successive intervals.
\end{abstract}

Recent research on the recognition of melodies has attempted to determine which cues are functional in recognition. Each note of a melody can be defined in terms of its fundamental frequency (tone height) as well as its note name, or tone chroma. The difference between the two formulations is that while tone height is defined on a unidimensional scale, the scale defining tone chroma can be conceptualized as two-dimensional and circular, since the identical note name is assigned to notes standing in an octave relation (i.e., a frequency ratio of 2:1) to one another (Shepard, 1964).

The validity of tone height as a description of perceiving periodic sounds has been well established by psychophysicists. Psychological scales of pitch corresponding to the physical scale of frequency have been constructed (e.g., Stevens, Volkmann, \& Newman, 1937). However, the validity of tone chroma is a bit less obvious, although evidence in support of tone chroma is compelling nonetheless. Two notes standing in an octave relation to one another may be perceived as more similar to each other than two notes which are closer in fundamental frequency. For example, conditioning studies using both animal and human subjects have found greater generalization for notes an octave apart than for notes whose frequencies have

This research was supported in part by National Institute of Mental Health Grant MH19399. Wendy Idson provided useful comments. Requests for reprints may be sent to either Howard J. Kallman or Dominic W. Massaro, Department of Psychology, University of Wisconsin, Madison, Wisconsin 53706. a ratio of somewhat less than 2:1 (Blackwell \& Schlosberg, 1943; Humphreys, 1939). Converging results are found when musicians are asked to judge the perceived similarity of tones of different frequencies (Allen, 1967).

Given these findings, one would expect tone chroma as well as tone height to play an important role in the recognition of melodies. To the extent that chroma is important, it should not matter much whether, for example, an $A$ note which follows a $_{4}$ (fundamental frequency of $392 \mathrm{~Hz}$ ) has a frequency of $440 \mathrm{~Hz}$ or, instead, $880 \mathrm{~Hz}$. However, Deutsch $(1969,1972)$ has suggested that although tone chroma is important for the perception of single tones, chroma plays no direct role in the recognition of melodies. Deutsch argues that the perception of melodies involves the abstraction of the frequency intervals separating temporally contiguous notes and that this abstraction process does not use chroma information. In support of this idea, Deutsch (1972) found that when the notes of the tune "Yankee Doodle" were each randomly displaced into one of three contiguous octaves, but tone chroma was preserved, recognition of the tune was extremely poor. It was reasoned that if tone chroma were important for the recognition process, recognition of the tune should have been easy.

One problem with Deutsch's conclusion is that her manipulation of tone height distorted the melodic contour of the original melody (Dowling \& Hollombe, 1977; Idson \& Massaro, 1978). That is, the up-down pattern of successive intervals was changed by randomly displacing the notes of the tune into the three 
octaves. In light of evidence implicating the importance of melodic contour information (Dowling, 1971; Idson \& Massaro, 1976), it is not unreasonable to expect recognition to suffer when the melodic contour of a melody is violated.

Idson and Massaro (1978) attempted to evaluate the relative roles of tone height, tone chroma, and melodic contour in melodic perception. In their experiments, subjects were asked to identify well-known tunes both in their original forms and after they had undergone systematic transformations. They found that when the sizes of the intervals in a tune were increased by one or more octaves, but the melodic contour was maintained (transformation OPC*octave, preserve contour), recognition was almost as good as when the original untransformed tune was presented. However, when the notes were displaced into different octaves without regard to contour (OVC*-octave, violate contour), recognition of the melody was poor. The finding of poor recognition, under the OVC* transformation essentially replicated Deutsch's result. Finally, when the contour of the melody was maintained but tone chroma was distorted (PC-preserve contour), performance suffered relative to the case in which both tone chroma and melodic contour were preserved. This latter finding suggests that the contribution of tone chroma to melody recognition cannot be reduced to the simple preservation of melodic contour.

The results of the Idson and Massaro study suggest that, contrary to Deutsch's view, tone chroma is important for the recognition of melodies. However, subjects in the Idson and Massaro experiments were given the names of the five test melodies and were then tested on these same melodies for hundreds of trials. It is possible that the subjects could have adopted strategies to recognize the melodies which would not be characteristic of how melodies are normally recognized. For example, Deutsch (1978) argues that listeners are able to use octave generalization to confirm the identity of a tune, but not to recognize it in the absence of prior information. This strategy would not require the use of successive interval information but might simply match each note against an auditory image of the relevant note of the hypothesized tune to determine if it is correct or stands in an octave relationship. Although this interpretation cannot easily explain the poor performance under the contour violating condition ( $\left.\mathrm{OVC}^{*}\right)$, it is possible that the elevated performance on transformation $\mathrm{OPC}^{*}$ relative to $\mathrm{OVC}^{*}$ was due to the use of strategies peculiar to the experimental task. For example, a hypothesis-testing strategy could depend on the melodies' having appropriate contours. Therefore, it seemed worthwhile to replicate the various transformations in a task in which these strategies could not be utilized. To this end, subjects in the present experiments were not informed in advance of the tune names, nor did they hear any given tune more than once. Thus the present experiments used manipulations similar to those used by Idson and Massaro, but minimized the degree to which special strategies could be responsible for the results.

\section{EXPERIMENT 1}

The first experiment contrasted the recognition of undistorted melodies with recognition of two octavedistorting transformations. One of the transformations (OVC) distorted the contour of the original melody, whereas the other transformation (OPC) left contour information intact (cf. Idson \& Massaro, 1978). Dowling and Hollombe (1977, Experiment 2) presented subjects with similar transformations, but in contrast to Idson and Massaro, they presented each melody to each subject a limited number of times. Subjects had difficulty in recognizing melodies that had undergone both octave and contour distortions. Recognition was better under the contourpreserving, octave-distorting condition, although performance given on some melodies was worse than it was for the original undistorted melodies. The melodies in the Dowling and Hollombe study were played on a piano and, as a result, each note consisted of a fundamental frequency plus upper harmonics. Given that the upper harmonics may have contributed to the role of tone chroma, the first experiment was an attempt to realize Dowling and Hollombe's basic findings using melodies composed of sine-wave tones.

\section{Method}

Subjects. Thirty-six subjects were recruited from the University of Wisconsin community and were paid 75 cents to participate for approximately $5 \mathrm{~min}$. Musical training was not a factor in their selection.

Stimuli and Apparatus. Three melodies were selected for use in the experiment: "Yankee Doodle," "London Bridge," and "Skip to My Lou." The notes of the original and transformed melodies appear in Table 1. Depending on the melody, the first 10-14 notes of the melody were used; rhythm information was generally maintained, although the rhythm of "Skip to My Lou" was simplified somewhat.

The OPC transformation involved increasing each successive interval in the melody by one octave while simultaneously preserving the original contour of the melody. On occasion, it was necessary to increase the interval's size by two or more octaves in order to keep all tones within the range of 150 to $4,000 \mathrm{~Hz}$. Intervals of unison in the original melody were maintained as unisons under transformation OPC. Transformation OVC was constructed without regard to the contour of the original melody, although chroma information remained intact under transformation OVC, as it did under OPC. Each note was randomly either higher or lower in frequency than the previous one, with the constraint that no note could fall outside the range $150-4,000 \mathrm{~Hz}$.

The sine-wave tones were produced by a Wavetek (Model 155) digitally controlled oscillator under the control of a program implemented on a PDP-8L computer. Since, for frequencies above 
Table 1

Note Names and Tone Frequencies of Melodies and Transformations Used in Experiments 1 and 2



Note-Note names for the original and two octave transformations appear above the tone frequencies. Note names for the PCS transformation appear below the tone frequencies. Quarter notes and eighth notes are denoted by upper-and lowercase letters, respectively.

$1,000 \mathrm{~Hz}$, the Wavetek oscillator could only be adjusted in increments of $10 \mathrm{~Hz}$, all note frequencies above $1,000 \mathrm{~Hz}$ were rounded to the nearest 10 s place. The output of the oscillator was gated by an Iconic (Model 0137) audio switch and was then recorded onto a Sony two-channel tape deck. The tones had rise and fall times of $10 \mathrm{msec}$. The duration of quarter notes, including rise and fall times, was $900 \mathrm{msec}$, and the duration of eighth notes was $400 \mathrm{msec}$. A 100 -msec silent interval separated successive tones.

During the experiment, the stimuli were played on the tape deck and amplified through a McIntosh (Model MC-50) amplifier. The tones were diotically presented to subjects over Grason-Stadler TDH -49 headphones at a level of approximately $82 \mathrm{~dB}(\mathrm{~A})$. Four or fewer subjects could be tested simultaneously in individual sound-attenuated rooms.

Procedure. All subjects heard the melodies in the same order: (1) "Yankee Doodle," (2) "London Bridge," and (3) "Skip to My Lou." The subjects were assigned randomly to one of three groups defined by which transformations were applied to the specific songs. Subjects in Group 1 heard the original undistorted version of "Yankee Doodle," followed by the OPC version of "London Bridge" and then the OVC version of "Skip to My Lou." Group 2 heard: "Yankee Doodle"-OPC, "London Bridge"-OVC, and "Skip to My Lou"-O (original). Completing the Latin square design, Group 3 heard: "Yankee Doodle"-OVC, "London Bridge"-O, and "Skip to My Lou"-OPC.
The subjects were told that they would hear three highly familiar melodies, but that some of the melodies had been "distorted" in various ways, making them difficult to recognize. The task was to write the name of each tune on an answer sheet. If the subjects knew some of the words but could not remember the tune's name, they were told to write down some of the words. Guessing was encouraged. Approximately $20 \mathrm{sec}$ separated presentations of the different melodies.

\section{Results and Discussion}

The percentages of correct identifications of each transformation of each melody appear in Table 2. Since the notes and rhythm of the portion of the song "London Bridge" which was presented to the subjects was almost identical to the notes and rhythm of the song "Mary had a Little Lamb," either response was scored as correct. All statistical tests were carried out using sign tests. ${ }^{1}$

Examination of Table 2 indicates that identification of the original undistorted melodies was quite good. Identification of the OVC transformations was much poorer than identification of OPC transformations $(p<.01)$. There was some decrement in performance

Table 2

Percentage of Correct Identifications of Each Transformation of Each of the Melodies in Experiments 1 and 2

\begin{tabular}{|c|c|c|c|c|c|c|c|c|}
\hline \multirow[b]{3}{*}{ Melody } & \multicolumn{8}{|c|}{ Transformation } \\
\hline & \multicolumn{4}{|c|}{ Experiment 1} & \multicolumn{4}{|c|}{ Experiment 2} \\
\hline & 0 & OPC & ovc & Mean & PCS & OPC & ovc & Mean \\
\hline $\begin{array}{l}\text { Yankee Doodle } \\
\text { London Bridge } \\
\text { Skip to my Lou }\end{array}$ & $\begin{array}{r}100 \\
92 \\
83\end{array}$ & $\begin{array}{l}83 \\
25 \\
75\end{array}$ & $\begin{array}{l}0 \\
8 \\
0\end{array}$ & $\begin{array}{l}61 \\
42 \\
53\end{array}$ & $\begin{array}{r}17 \\
17 \\
0\end{array}$ & $\begin{array}{l}92 \\
58 \\
67\end{array}$ & $\begin{array}{r}8 \\
17 \\
0\end{array}$ & $\begin{array}{l}39 \\
31 \\
22\end{array}$ \\
\hline Mean & 92 & 61 & 3 & 52 & 11 & 72 & 8 & 31 \\
\hline
\end{tabular}


on transformation OPC ( $\mathrm{p}<.01)$ but this was only substantial for "London Bridge." These results are in essential agreement with the findings of Dowling and Hollombe. In their study, however, identification of the tunes "Mary had a Little Lamb" and "Yankee Doodle" averaged around $40 \%$ on an OVC transformation. In contrast, there were virtually no correct identifications of the OVC transformations in the present study. The better performance in the Dowling and Hollombe study on the OVC transformation may have been due to the fact that piano sounds rather than sine-wave tones were used; the presence of the upper harmonics might compensate, in part, for the fact that the contour of the fundamental frequencies was incorrect. If the contour were less well defined when upper harmonics accompany the fundamental, violation of fundamental frequency contour would have less of an effect.

Although the results of the first experiment and the study of Dowling and Hollombe (1977) establish the importance of melodic contour, it is not clear whether the relatively good performance on transformation OPC was due entirely to the utilization of contour information or whether, instead, tone chroma also contributed to recognition of the melodies. In order to evaluate the contribution of contour, it is necessary to preserve a similar amount of contour information without chroma information and contrast performance with performance on the OPC transformation. This contrast was carried out in Experiment 2.

\section{EXPERIMENT 2}

\begin{abstract}
Method
The procedure of Experiment 2 was virtually identical to that of Experiment 1, except that a new transformation-PCS (preserve contour stretched)-was used in place of the original undistorted melodies used in Experiment 1 (cf. Idson \& Massaro, 1978). The OPC and OVC transformations of Experiment 1 were also tested in Experiment 2. Counterbalancing of melody presentations was as in Experiment 1, except that the PCS transformation substituted for the original $(O)$ melodies. Thirty-six new subjects were recruited and paid 75 cents each.

The PCS transformations were derived from the OPC transformations (see Table 1). To arrive at the PCS transformation, each note of the OPC transformation, with the exception of the first note of each melody, was randomly raised or lowered one or two semitones, with the restriction that none of the successive intervals of the PCS transformation, with the exception of unison, could be the same size as the corresponding OPC interval. The PCS transformation thus maintained correct contour information while eliminating accurate tone chroma. If the identification of OPC transformations in Experiment 1 were based solely on contour information, performance equal to that on transformation OPC should be found on transformation PCS. If chroma also played a critical role, however, performance on transformation PCS should be worse than on OPC.
\end{abstract}

\section{Results and Discussion}

The data from Experiment 2 are presented in Table 2. Subjects rarely identified the OVC trans- formations but did quite well on the OPC transformations $(p<.01)$. Of particular interest, performance on transformation PCS was significantly poorer than performance on OPC $(\mathrm{p}<.01)$ and not significantly better than performance on transformation OVC $(p>.10)$. The results suggest that the relatively good performance on transformation OPC depends on both accurate contour and chroma information.

\section{GENERAL DISCUSSION}

The present experiments strongly implicate the importance of melodic contour in melody recognition, as the poor performance on transformation OVC indicates. The poor performance on the PCS transformation in Experiment 2 suggests that contour information alone is not sufficient for accurate melody recognition. Both chroma and contour information are necessary for anything approaching accurate identification of melodies. However, tone height has some importance in that the original melodies are more easily recognized than transformation $O P C$.

It is difficult to interpret the differences that were found for the three different melodies. It appears that the original melodies were not equally difficult, although the extremely good performance prevents a reliable assessment of any differences. The most striking difference was the poorer performance on transformation OPC of "London Bridge" than on the other two melodies, especially in Experiment 1. It might be that the good performance on transformation OPC for "Yankee Doodle" and "Skip to my Lou" was due to the unison intervals that were preserved in transformation OPC. "London Bridge" does not have a unison interval and, therefore, transformation OPC would be more difficult. However, "Skip to my Lou" has two unison intervals and "Yankee Doodle" just one, and yet the two melodies did not appear to be differentially affected by transformation OPC. Furthermore, transformation PCS also preserved the unison intervals of the original melodies, and performance on this transformation was equally bad for the three melodies. One might expect good performance on transformation PCS of melodies with unison intervals if the unison intervals aid performance on transformation OPC. Although this issue cannot presently be resolved, the differences in performance across the three melodies to not weaken the conclusions that have been derived.

The results of the present experiments replicate those of Idson and Massaro (1978) by showing that both chroma and contour information contribute to melody recognition. Therefore, it cannot be argued that Idson and Massaro's (1978) results are unique to the forced-choice procedure with repeated observations. The hypothesis-testing strategy proposed by Deutsch (1978) is not a necessary condition for a 
functional role of tone chroma in melody recognition.

The results of Idson and Massaro (1978) and the present study show that chroma information is used in the recognition of melodies. However, they do not necessarily argue against Deutsch's (1969) suggestion that the abstraction of successive intervals is fundamental to melody recognition. Octave generalization could also operate in the perception of successive intervals. According to this view, the perceived similarity of successive intervals would be analogous to the perceived similarity of individual tones. Single tones differing by exactly an octave are perceived as more similar than tones differing by slightly more or less than an octave. Successive intervals differing by exactly an octave would be perceived as more similar than successive intervals differing by slightly more or less than an octave (Thurlow \& Erchul, 1977). Octave generalization would allow an interval of, for example, an ascending ninth (a major second plus an octave) to be perceived as highly similar to an ascending major second. Interpreting the interval as an ascending major second would be quite appropriate, given that intervals greater than an octave are rarely found in melodies. This interpretation also predicts the poor performance on transformation OVC. If contour is violated, a successive interval in the transformed melody will always differ by less than an octave from the original interval. For example, transforming a descending minor seventh by an octave will produce an ascending major second when contour is violated. The transformed interval of an ascending major second would not be interpreted as a descending minor seventh, since these two intervals are not perceived as similar to one another. This view is not only consistent with the results of melody recognition experiments, it has the appealing property of following naturally from the phenomenon of octave generalization.

\section{REFERENCES}

Allen, D. Octave discriminability of musical and non-musical subjects. Psychonomic Science, 1967, 7, 421-422.

Blackwell, H. R., \& Schlosberg, H. Octave generalization, pitch discrimination, and loudness thresholds in the white rat. Journal of Experimental Psychology, 1943, 33, 407-419.

Deutsch, D. Music recognition. Psychological Review, 1969, 76, 300-307.

Deutsch, D. Octave generalization and tune recognition. Perception \& Psychophysics, 1972, 11, 411-412.

DeuTsch, D. Octave generalization and melody identification. Perception \& Psychophysics, 1978, 23, 91-92.

Dowling, W. J. Recognition of inversions of melodies and melodic contour. Perception \& Psychophysics, 1971, 9, 348-349.

Dowling, W. J., \& Hollombe, A. W. The perception of melodies distorted by splitting into several octaves: Effects of increasing proximity and melodic contour. Perception \& Psychophysics, 1977, 21, 60-64.

Humphreys, L. G. Generalization as a function of method of reinforcement. Journal of Experimental Psychology, 1939, 25, 361-372.

IDSON, W. L., \& Massaro, D. W. Cross-octave masking of single tones and musical sequences: The effects of structure on auditory recognition. Perception \& Psychophysics, 1976, 19, 155-175.

IDSON, W. L., \& MASSARO, D. W. A bidimensional model of pitch in the recognition of melodies. Perception \& Psychophysics, 1978, 24, 551-565.

ShEPARD, R. N. Circularity of judgments of relative pitch. Journal of the Acoustical Society of America, 1964, 36, 2346-2353.

Stevens, S. S., Volkmann, J., \& Newman, E. G. A scale for the measurement of the psychological magnitude of pitch. Journal of the Acoustical Society of America, 1937, 8, 185-190.

Thurlow, W. R., \& Erchul, W. P. Judged similarity in pitch of octave multiples. Perception \& Psychophysics, 1977, 22, 177-182.

\section{NOTE}

1. In conducting the sign test, melody as a factor was disregarded. For example, a subject's response on the original version of "Yankee Doodle" was compared with his/her response on the OPC transformation of "London Bridge." The counterbalancing scheme should have minimized any biases that this introduced.

(Received for publication October 30, 1978; revision accepted March 30, 1979.) 